\title{
QUALIE ACOUSTIQUE DE QUELQUES SALLES DE CONCERT EN ALLEMAGNE
}

\author{
C. SEMIDOR-SIGNORET, P. JOUENNE*, C. BONHOMME et P. JOANNE
}

Labo C Ecole d'Architecture de Bordeaux, Domaine de Raba, F-33405 Talence cedex, France

*Université Paris VI, Laboratoire de Mécanique Physique, Unité de Recherche Associée au CNRS $N^{\circ} 868$, "Acoustique et Mécanique", Centre de Recheche et Transfert de Technologie, Acoustique Architecturale et Urbaine, Le Val Joyeux/CD 11, F-78450 Villepreux, France

Here we propose a comparative study of the acoustic quality of three concert halls in Germany. The results of the acoustic measurements are optimized by comparing them with the reactions of musiciens who have performed in these halls. The acoustic parameters being analyzed are the TR60, Clarity, EDT, "pouvoir separateur" (that is to say the determination of length of time between two separate sounds), and background noise. The bringing together of these parameters and the subjective opinions of the musiciens coincide in the final analysis.

\section{1 - Introduction}

Cette communication porte sur la comparaison des qualités acoustiques de trois salles de spectacle situées en Allemagne à partir, d'une part des résultats des mesures acoustiques réalisées selon un protocole mis au point au laboratoire d'Acoustique et de Mécanique URA868 [1], et d'autre part des opinions émises par des musiciens de l'Orchestre National de Bordeaux Aquitaine, que les auteurs ont suivi lors d'une de ses tournées. Les résultats, présentés ici, font partie d'un corpus portant sur une dizaine de salles d'Allemagne et d'Autriche testées lors de cette tournée.

On remarque sur le plan et la coupe de chacune des salles, que leur architecture est très différente, aussi bien en forme qu'en volume.

a Aachen
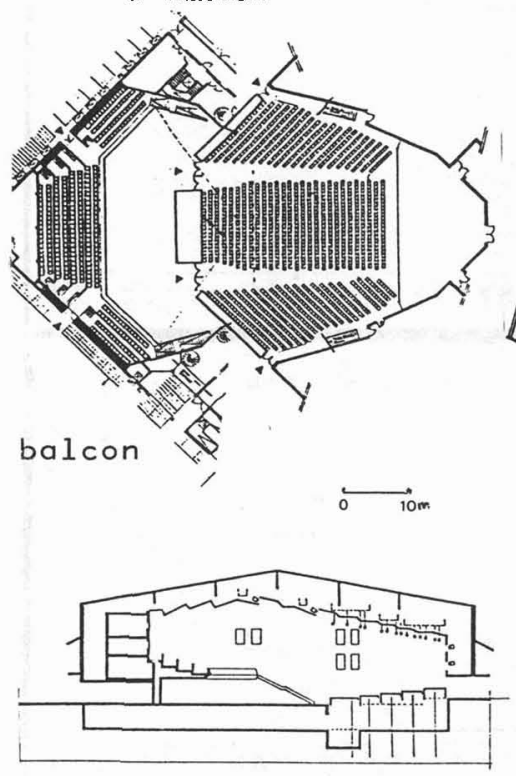

b Berlin
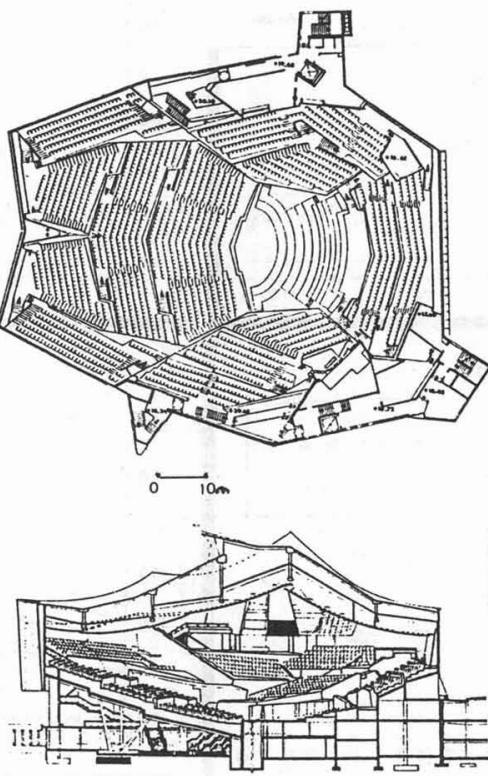

c Leverkusen
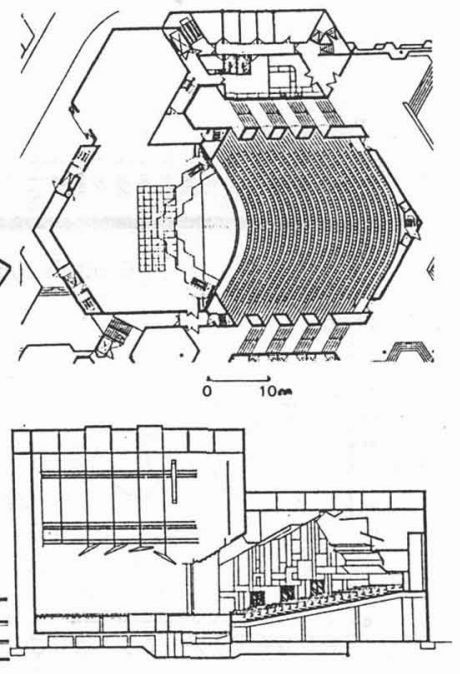


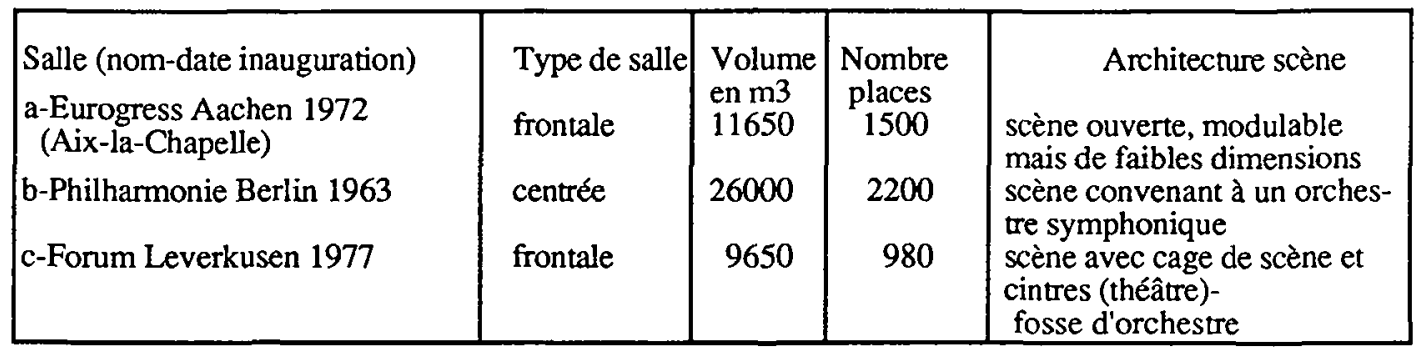

\section{2 - Protocole de mesures et analyse des données acoustiques}

Le principe des mesures en salle est le suivant : on dispose une source sonore (générateur de bruit rose amplifié) en des points différents de la scène (de 1 à 5) et on relève plusieurs points d'écoute (de 3 à plusieurs dizaines) dans la salle le signal reçu au moyen d'un magnétophone numérique autorisant une grande plage dynamique à l'enregistrement. Le dépouillement se fait en laboratoire de façon automatique grâce aux techniques récentes d'acquisition et de traitement du signal numériques.

Les signaux, échantillonnés à $48 \mathrm{kHz}$ avec une résolution de 16 bits par le magnétophone numérique, sont transmis à un ordinateur équipé de cartes de traitement du signal. Après filtrage, les spectres composant la séquence sont obtenus sous la forme de 27 tiers d'octave de $50 \mathrm{~Hz}$ à $20 \mathrm{kHz}$. L'acquisition simultanée des spectres permet la comparaison précise des phénomènes temporels, en particulier la détermination des instants de déclenchement et d'extinction de la source sonore.

La modélisation et le calcul des paramètres se font par le logiciel SALLE [1]. Parmi les paramètres acoustiques accessibles par cette analyse, on détermine : TR60, EDT [2], Clarity, le pouvoir séparateur et le spectre de bruit de fond.

Pour chaque salle une trentaine de séquences correspondant à un couple (source-écoute) sont traitées. Le tracé des diagrammes donne une synthèse de la qualité acoustique du lieu testé.

Les résultats présentés sont obtenus après un moyennage des données sur :

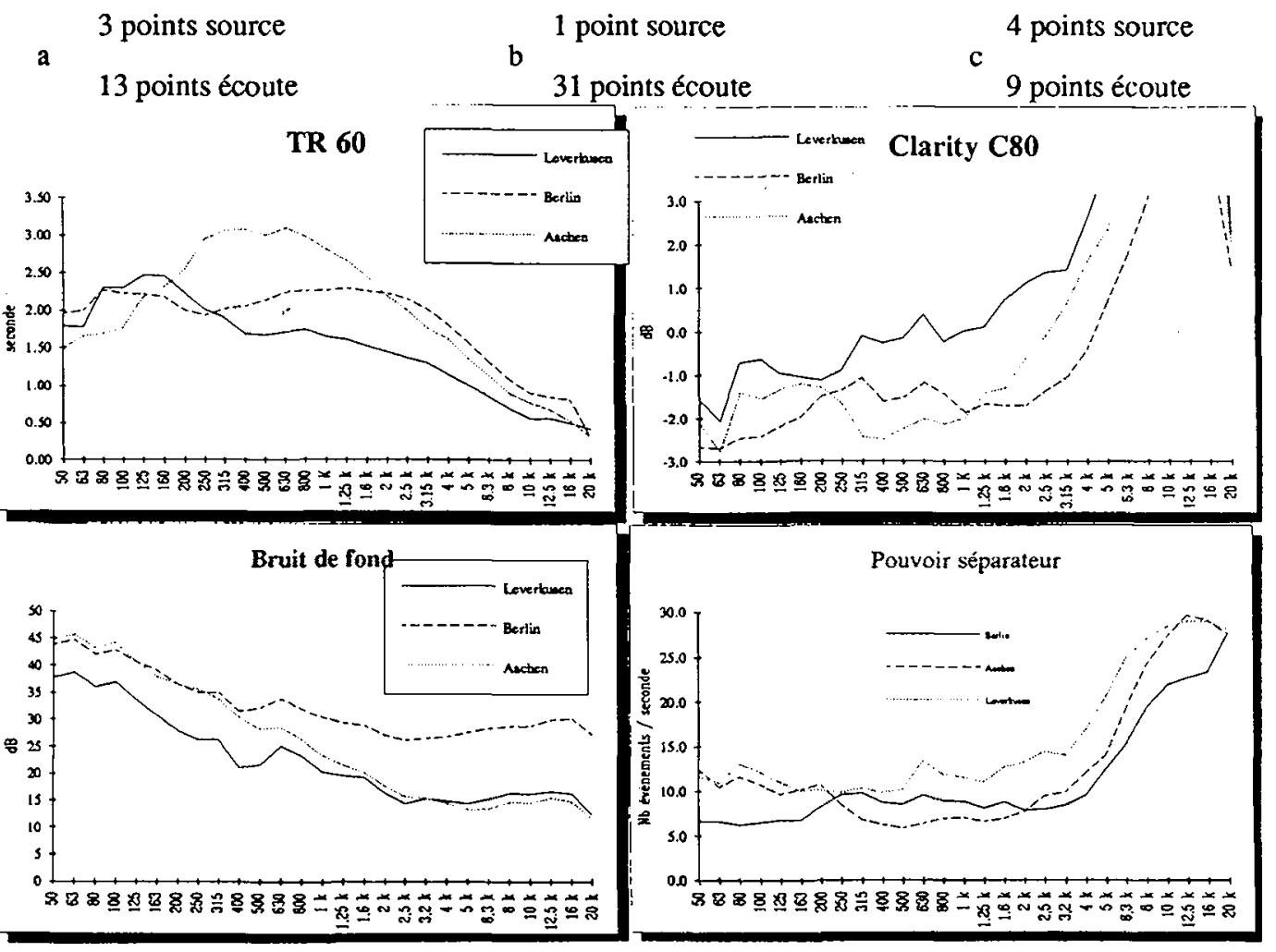



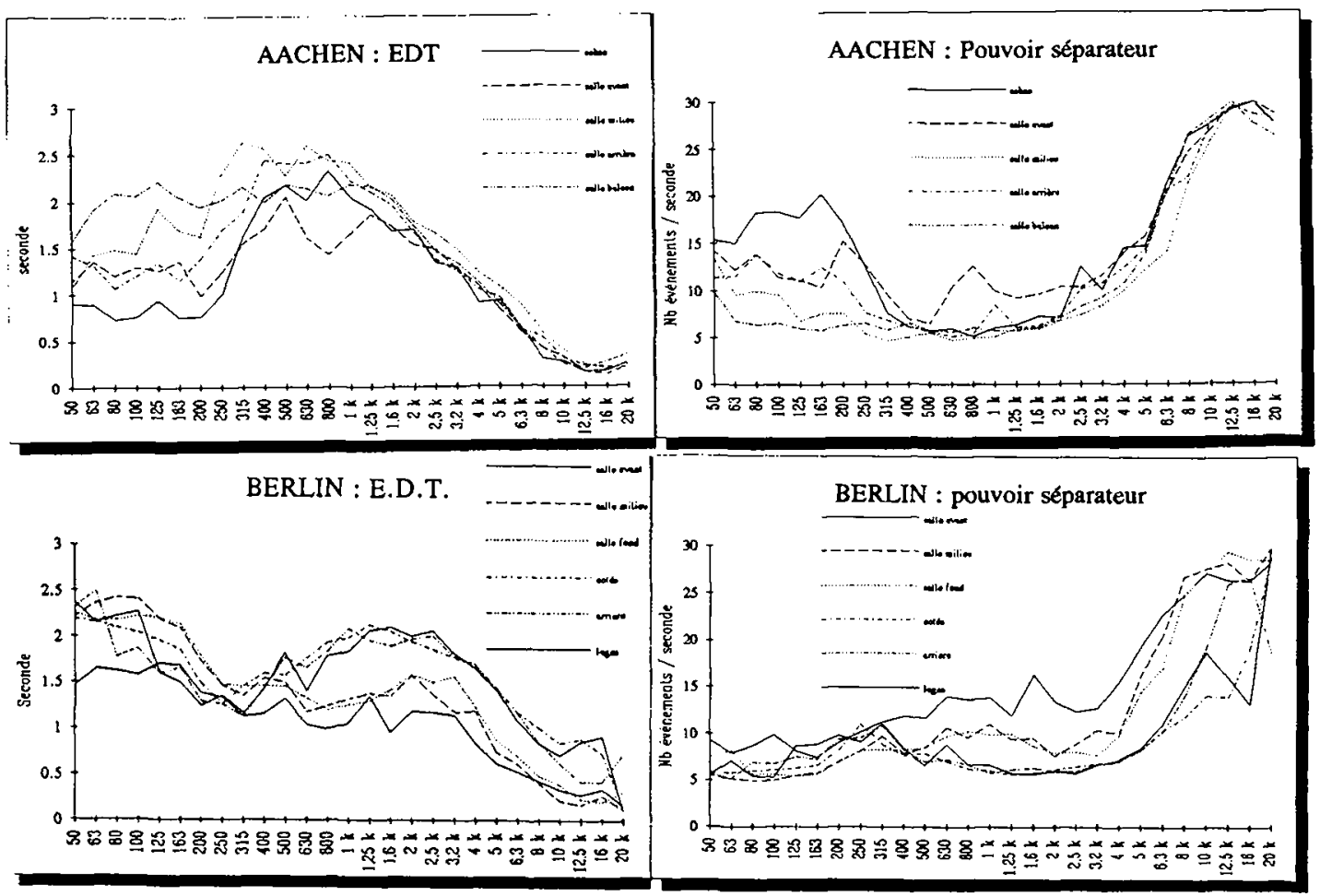

LEVERKUSEN : EDT
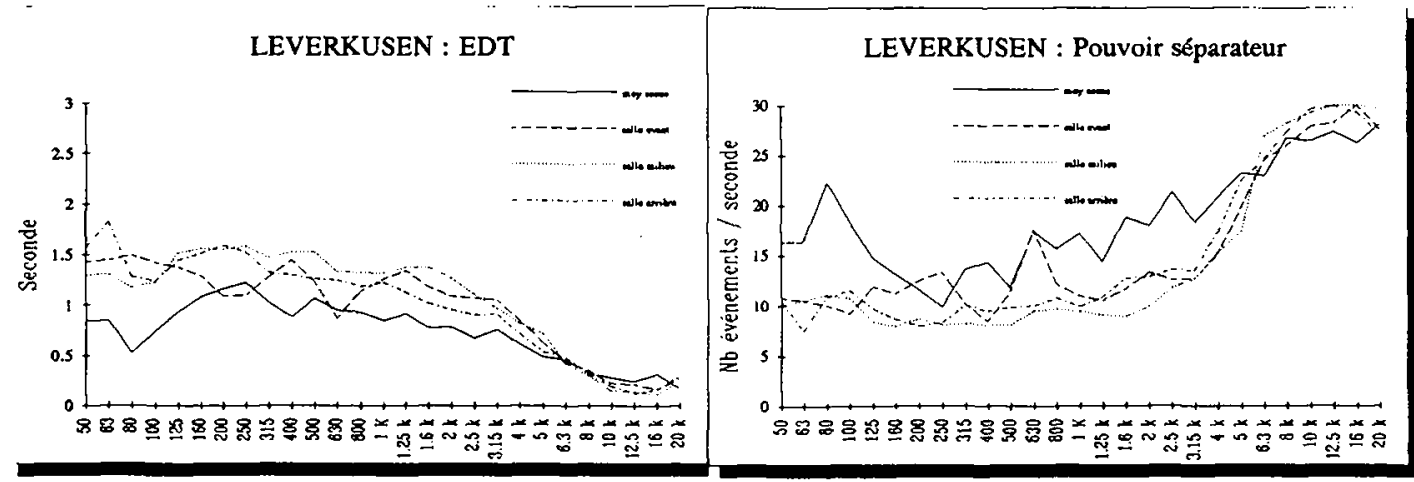

\section{3 - Résultats de l'enquête}

On a demandé aux musiciens qui ont accepté de collaborer à cette étude de remplir après chaque concert un questionnaire type concernant la salle dans laquelle ils venaient de se produire. Ces questionnaires regroupés dans un dossier remis à chaque volontaire au début de la tournée, comportent treize questions, formulées après une discussion préalable avec les musiciens. Pour chaque salle suit une enquête portant sur l'appréciation générale tant du point de vue de l'acoustique que du confort : ambiance intérieure (couleur, lumière, chaleur,...) architecture (forme, matériaux, espace sur scène, loges,...)

Les réponses présentées sous forme d'histogrammes sont relatives aux questions permettant une comparaison avec les paramètres acoustiques analysés. On propose au choix quatre niveaux de réponse : Bien (A), Moyennement (B), Un peu (C), Pas du tout (D). 
Percevez-vous clairement et distinctement

- le (ou les) soliste (s)
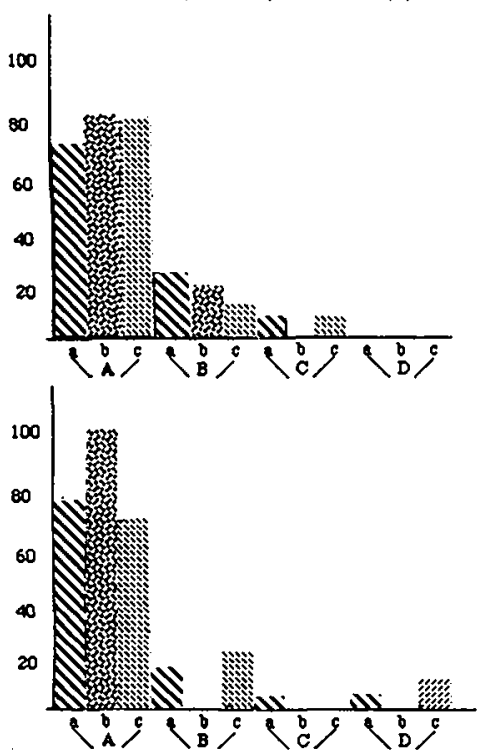

- Avez-vous l'impression que le son passe vers la salle?

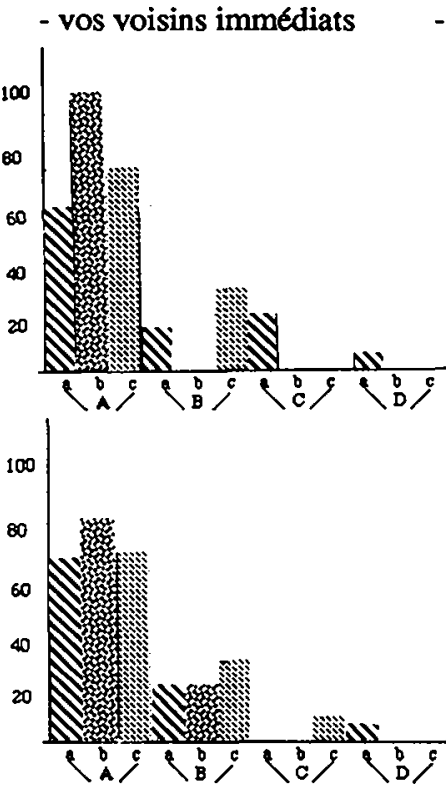

- les musiciens les plus éloignés

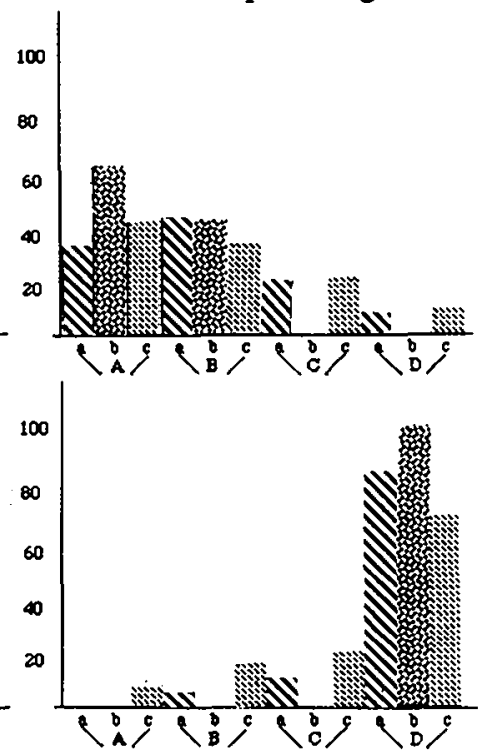

- L'attaque des sons vous semble-t-elle franche?
- Etes-vous gêné par des bruits qui vous masquent l'orchestre

L'ensemble des pupitres est représenté dans la population ayant accepté de remplir un dossier. Voici, extraits des dossiers des musiciens les avis sur chacune des salles avec le vocabulaire qui leur est propre :

a : cette salle vide est très réverbérante - moins quand elle est pleine - cet espace est très "résonant" - le volume est trop sonore - le son est confus dans les fortissimo-les timbres sont mal respectés -

$\mathrm{b}$ : il est agréable de jouer dans cette salle - le son est plutôt brillant et chaud, sans halo sonore - sa coloration est correcte - la salle donne une sensation d'intimité et semble vivante -

$\mathrm{c}:$ le son est feutré comme dans un amphithédtre, la sonorité dure et sèche - il faut jouer fort pour faire passer le son vers la salle - la scène d l'italienne oblige a suivre le chef d l'oeil -

\section{4 - Conclusion}

On peut noter un bon recoupement concernant les avis des musiciens et les paramètres acoustiques déterminés, pour chacune des salles.

A Aachen EDT passe par une valeur maximale de 2,5s dans les médiums et décroît très vite dans les graves et les aigus ce qui concorde avec les remarques des musiciens sur l'espace "résonant" et une confusion du son ressentie dans la zone de plus grande sensibilité de l'oreille.

A Berlin les valeurs maximales de EDT dans le haut médium (2s) et dans les graves $(2,5 \mathrm{~s})$ expliquent qu'ils aient trouvé le son plutôt brillant et chaud.

A Leverkusen les valeurs relativement faibles de EDT sur tout le spectre peuvent justifier que la sonorité ait été ressentie comme dure et sèche.

[1] P. JOUENNE actes du colloque "La qualite sonore des espaces habités" Grenoble mars 1991 [2] V.L. JORDAN JASA 47, 1081970

Que les musiciens de l'ONBA, son directeur et son administrateur, reçoivent tous nos remerciements pour l'aide qu'ils ont bien voulu nous apporter. 